\title{
El Votanero: creación y evaluación de un portal informativo sobre las elecciones presidenciales en México $2018^{*}$
}

\author{
Zanely Alicia Castro Guerrero ${ }^{1}$, Alejandra Sarahy Cavazos Pompa ${ }^{2}$, María Fernanda Lau \\ García $^{3}$, Nohemí Vilchis Treviño ${ }^{4}$
}

\begin{abstract}
Resumen
Al percibir la ausencia de plataformas digitales confiables que generaran contenido sobre las elecciones presidenciales méxico 2018 y, en particular, información de los candidatos involucrados en la contienda, se concibió la idea de crear un portal informativo en Facebook: el VOTAnero. Con esta iniciativa se buscó facilitar la información para que los usuarios mexicanos emitieran un voto a consciencia y a partir de los datos recolectados se realizó una evaluación en cuanto a la utilidad, percepciones de neutralidad de los seguidores y su comportamiento. Después de recabar los hallazgos, la neutralidad no fue percatada por algunos usuarios.

Abstract

Upon perceiving the absence of reliable digital platforms that generate content about Presidential Elections in Mexico 2018 and, in particular, information from the candidates involved in the contest, the idea of creating a revealing portal on Facebook was conceived: EI VOTAnero. This initiative sought to provide information for mexican users to cast a vote in conscience and based on the data collected, an evaluation was made regarding the utility, perceptions of neutrality of the followers and their behavior. After collecting the findings, neutrality was not noticed by some users.
\end{abstract}

Palabras Clave

México, Elecciones, Candidatos, Redes sociales, Partidos políticos.

Keywords

Mexico, Elections, Candidates, Social networks, Political parties.

'Segundo lugar del concurso de Trabajos Recepcionales en Comunicación CONEICC 2019. Universidad de Monterrey. Asesora de tesis: Dra. Beatriz Elena Inzunza Acedo.

1Autor para correspondencia: nohemi.vilchis@udem.edu

\section{Introducción}

La época electoral en México es un conjunto de matices en donde se combinan candidatos, publicidad, marketing y campañas políticas. Los electores caen en desinformación y son víctimas de mensajes ambiguos, lo cual crea que se efectúen votos sin sustento por costumbre, conveniencia o inclusive con base en sobornos (a manera de despensas, promesas, viviendas, etcétera) (La Jornada, 2017).
La ciudadanía vota pero no siempre hay una participación activa. Ante esto, la democracia pierde sentido y legitimidad dado que los niveles de participación son insuficientes. Las razones son que no existe interés del ciudadano o de aquellos que lo rodean, la ausencia de eficacia política al considerar que la opinión individual no es importante y la falta de información. Los mexicanos están lejos de la política porque solo ven corrupción, misma que produce desconfianza (Cruz, 2016). 
Vázquez (2017) menciona que en un estudio realizado por Freedom House se identificó que México tiene una de las más altas tasas de penetración de redes sociales en el mundo. También se observó que las fake news, los bots, influencers pagados, restricciones de internet y el robo de información personal cuestionan la capacidad del internet para generar espacios incluyentes y libres de diálogo que conforman los pilares de la democracia. Debido a esto, es necesario crear plataformas que permitan la exposición de mensajes neutrales con el fin de que el usuario se beneficie de la manera que más le convenga.

Por consiguiente, se optó por desarrollar un proyecto al que se le llamó "El Votanero", el cual constó de la creación de una página a través de la red social Facebook donde se divulgó información política sobre los candidatos a la presidencia así como el proceso electoral de México 2018. Una vez terminada la temporada de elecciones, se hizo una evaluación de la misma.

\section{Antecedentes}

El Estudio Censal de la Participación Ciudadana (2013) indicó que durante la jornada electoral de julio 2012 la participación ciudadana alcanzó un $62.08 \%$ de la lista nominal, lo que representó 3.85 puntos porcentuales más que las cifras del 2006. Es decir, hubo una mayor asistencia a las urnas electorales en 2012 que en 2006. Sin embargo, los números no superan el máximo histórico de 1994 con $77.16 \%$ (IFE, 2013).

Cruz (2016) menciona que el desarrollo de la democracia en México ha ido perdiendo valor y credibilidad. Esto lo atribuye a los problemas de corrupción en los más altos niveles del gobierno en el país. Sin embargo, el narcotráfico, la violencia e inseguridad también han sido causas de la regresión en el tema.

El informe País sobre la Calidad de la Ciudadanía en México realizado en 2014 (Somuano y Nieto, s.f.) definió que el número de mexicanos que ejercen una participación política distinta al voto, es pequeño. El estudio determinó que los mexicanos entienden el concepto de ciudadanía como una materia de subordinación de las leyes y el voto, que está separado de la noción de participación, organización colectiva o de ejercer derechos.
Serrano (2015) indica que los ciudadanos no colaboran en temáticas de interés general principalmente porque no tienen la información necesaria para evaluar al gobierno o para coadyuvar en programas y políticas. Igualmente, la baja participación se debe a la falta de confianza en las instituciones, la ausencia de transparencia o rendición de cuentas y a causa de las violaciones hacia las garantías individuales.

Asimismo, otro obstáculo de la realidad social actual es el auge de las fake news que generan desinformación en redes sociales, pues Elósegui (2017) menciona que las noticias falsas viajan más rápido de lo que pueden ser desmentidas. También describe que en la actualidad las fake news en materia de política son las que más preocupan, ya que se desconoce su influencia en las votaciones y en el imaginario de la ciudadanía.

Algunas de las iniciativas realizadas anteriormente incluyen la efectuada por la Universidad Nacional Autónoma de México (UNAM), misma que lanzó una plataforma en el 2015 llamada "Voto Informado" para alentar a los ciudadanos a que emitieran precisamente un voto a conciencia, en las elecciones que tuvieron presencia el 7 de junio de ese mismo año en las contiendas electorales federales. Fernando Castañeda Sabido (2015), director de la Facultad de Ciencias Políticas y Sociales comentó que el proyecto es apartidista y no tiene preferencia ni ideología alguna. La página funcionaba de la siguiente manera: los partidos o sus representantes contestan con sus propuestas una serie de cuestionarios de diferentes temas y responden dudas comunes, esto toma alrededor de 15 minutos y se hace por medio de bloques, la base de datos es pública y el portal se mantiene abierto hasta horas antes del cierre de campaña. De los datos del año 2015, se obtuvo que aproximadamente 1,084 candidatos a la diputación federal se inscribieron a la plataforma.

Campaigns and Elections (2012) inició en Estados Unidos como una revista enfocada en campañas políticas con base en herramientas, tácticas y técnicas de la consultoría política que, hasta hoy en día, cuenta con ediciones en México y Canadá. Dentro de su página, existe un apartado donde se lee Candidate File que da la oportunidad de consultar datos relevantes acerca de los candidatos a la elección. Por otro lado, la página de inicio del portal en México contiene noticias electorales, vídeos y memes en referencia 
a los candidatos a presidencia México 2018. De acuerdo a una nota publicada por Nyczepir (2012), Generation Opportunity, una organización activista estadounidense dirigida a jóvenes adultos, realizó una encuesta donde sus resultados concluyeron que jóvenes millennials prefieren Facebook y mensajes de textos como los principales medios para recibir información con respecto a las elecciones. También, la tercera herramienta que esta generación prefiere es el correo electrónico.

\section{Objetivo general}

Crear un portal informativo que promueva un voto con conciencia de los candidatos a la presidencia México 2018 durante la campaña electoral y, con los datos recolectados, realizar una evaluación del mismo.

\section{Objetivos específicos}

- Realizar publicaciones neutrales con fuentes fidedignas a fin de informar a los seguidores de la plataforma en los temas de trayectoria de los candidatos presidenciales, sus propuestas y datos prácticos sobre el proceso electoral. Formato utilizado: infográficos.

- Hacer una evaluación del portal en cuanto a la utilidad y percepciones de neutralidad de sus seguidores, así como el comportamiento de usuarios en Facebook, por medio de la plataforma Netvizz, las estadísticas de Facebook Analytics y la aplicación de dos encuestas.

\section{Justificación}

En vista de los constantes bombardeos de información falsa, fue conveniente crear un portal que incitara a los usuarios de Facebook a estar conscientes de la jornada electoral y al mismo tiempo ofrecer neutralidad al informar sobre los candidatos para la presidencia de México. Por ello, "El VOTAnero" nació con el propósito de contrarrestar la desinformación que pueda repercutir o influir en la decisión del votante.

Además, la información recabada en los infográficos expuestos en el portal informativo facilitó el proceso de investigación, por el que debería atravesar el votante que tiene en sus manos el derecho de escoger al candidato de su preferencia para representar al país.

A pesar de que este trabajo no erradica la problemática de la desinformación por completo, sus implicaciones pueden ayudar como precedente para futuros proyectos similares que busquen beneficiar al electorado a través de plataformas políticas en línea y en conjunto resuelvan una amplia gama de problemas prácticos. Como valor teórico, la evaluación del portal puede funcionar para que se realicen investigaciones que otorguen validez para seguir desarrollando este tipo de temas y así crear teorías sobre el votante mexicano junto con otras variables.

\section{Proyecto EI VOTAnero}

Después de un análisis y, dado el limitado tiempo de las campañas presidenciales (30 de marzo a 27 de junio de 2018), se optó por Facebook como plataforma única gracias a los diferentes públicos que se encuentran suscritos en ella, específicamente 83 millones de mexicanos activos al mes (Hootsuite y We Are Social, 2018) en comparación con Twitter que solo cuenta con cuatro millones (Islas, 2018). Una vez elegida la red social, se decidió solamente utilizar infográficos concretos y específicos como vía de información.

En cuanto al diseño, su composición se creó adaptándose específicamente a la audiencia, de modo que le permitiera ser llamativo y dinámico, además de contener colores que no se asociaran con los partidos contendientes del momento, las identidades gráficas de los candidatos independientes o alguna de las 18 Secretarías. Resultando en la utilización del pantone P 14-7C, negro y blanco, así como la tipografía "Montserrat".

También se ilustraron íconos con los rostros de los candidatos a la presidencia México 2018, siguiendo los rasgos más significativos de cada uno para el fácil reconocimiento del usuario. El concepto gráfico para la imagen que se quería lograr, debía tener un balance entre lo llamativo y lo formal. Por tanto, se decidió llegar a esto, por medio de infográficos con textos breves pero que contaran con información adaptada al lenguaje coloquial mexicano.

De igual forma, se llegó al acuerdo de que no se revelaría la identidad de los administradores, sino que se hablaría como un grupo de personas preocupadas por la desinformación de los usuarios en busca de que la ciudadanía emitiera un voto 
informado en las elecciones presidenciales México 2018, sin comprometer la neutralidad a través del sesgo. A su vez se optó por no responder a los comentarios de los usuarios por las mismas razones.

Las publicaciones fueron divididas por secciones según la temática y objetivo. La primera fue “Sabías qué?”, misma que se realizó con el objetivo de adquirir los primeros seguidores de la página, al compartir información general sobre el proceso electoral, la cultura de la legalidad, los candidatos seleccionados, sus coaliciones correspondientes, entre otras.

La segunda sección fue lanzada el 31 de marzo de 2018, llamada "Trayectorias", donde se expusieron los datos generales de cada uno de los candidatos como: su edad, lugar de nacimiento, estado civil, su grado de estudios y la experiencia que tienen en distintos cargos públicos.

Más adelante, se publicaron las propuestas de los candidatos con respecto a cada uno de los temas más relevantes de las 18 Secretarías que conforman la Federación: corrupción, educación, política-electoral, seguridad, salud, empleos y salario mínimo, medio ambiente, vivienda, turismo, pobreza, valores, grupos vulnerables, transporte, cultura, desarrollo rural, energía, economía y política exterior.

Otra de las secciones fue "Días de", con espacios dedicados específicamente a los candidatos. El contenido fue separado en cuatro imágenes que proporcionalmente formaban una misma, publicadas en orden alfabético y diferentes días. En ellas se incluyeron las temáticas incorporadas en sus propuestas, datos del personaje, declaraciones, presentación de gabinete, coordinadores de campaña y redes sociales.

Finalmente se difundieron las publicaciones del 1 de julio. Antes del día de la jornada electoral, se reiteraron las cuestiones básicas para emitir un voto informado. Cuando se reveló al candidato que el 1 de diciembre tomara protesta como presidente de México, "El VOTAnero" informó de su triunfo y recordó las propuestas a fin de que la ciudadanía exija lo que prometió realizar durante su gestión.

\section{Evaluación}

Se realizó una evaluación del portal informativo de “El VOTAnero" con el objetivo de saber qué tanto estuvieron al pendiente los 3,245 seguidores de la página, el tipo de comentarios que se realizaron en cada una de las publicaciones, los mensajes que escribían en las infografías compartidas en sus perfiles, así como sus reacciones y sugerencias del contenido.

Después de que se eligió a un candidato electo para la presidencia de México, el 5 de julio de 2018 se publicó una encuesta realizada a través de Google Forms para que los seguidores del portal informativo emitieran su opinión sobre el trabajo de la iniciativa. Sin embargo, como en la primera encuesta, la muestra no fue representativa y para obtener resultados más precisos, se decidió crear un segundo sondeo, utilizando la misma plataforma.

Con este método, algunos de los resultados arrojados fueron que en cuanto a si la información que se compartió en la página se consideraba neutral, se obtuvo que un $81.3 \%$ de los encuestados respondió que sí, el $10.9 \%$ seleccionaron que había sesgo hacia el candidato Andrés Manuel López Obrador, un $5.5 \%$ dijo que existía sesgo hacia el candidato José Antonio Meade Kuribreña y un $2.3 \%$ respondió que había sesgo hacia el candidato Ricardo Anaya Cortés.

Con el fin de profundizar en los hallazgos se realizaron tablas de contingencia en el programa de IBM SPSS, un software de análisis que ofrece recolección de datos y comparación de los mismos el cual es fácil de usar, para correlacionar distintas preguntas y sus variables. Dentro de ellas se arrojaron distintos resultados.

La tabla de contingencia con las preguntas "no expresé mis inclinaciones políticas por temor a la opinión de la mayoría" y "estoy de acuerdo con la frase "de política y religión no se debe hablar' revela que los seguidores que sí expresaron sus inclinaciones políticas ante la sociedad y no están conformes con la frase, son pocos, pues por lo general sí tienen miedo de manifestar sus ideales porque apoyan el dicho popular.

Análogamente, la comparativa entre la edad de los encuestados y si "conocí la mayoría de las propuestas de todos los candidatos" arrojó que los jóvenes de 18 a 29 años son los que se consideran más informados sobre las propuestas de los candidatos a la presidencia. Los seguidores de 46 a 65 años también se consideran informados sobre lo que proponía cada candidato. 
La tabla de contingencia con las variables "qué tipo de información compartiste más" y si "compartí información de las elecciones en redes sociales con el fin de convencer a mis seguidores a votar por alguien" respondieron informar a su red de contactos para convencerlos a través de redes sociales y burlas/memes, realizando así una anti-campaña hacia los candidatos que no eran de su preferencia.

También se incluyó a Netvizz como uno de los métodos de análisis por su facilidad de acceso. La anterior, es definida como una aplicación desarrollada para funcionar a través de Facebook como un extractor de datos de diferentes secciones de la red social, ya sea en perfiles personales, grupos y páginas para fines de investigación (Benítez, 2014). Los archivos resultantes pueden analizarse fácilmente a través de softwares estándar como Excel, Guess o Gephi (Iniciativa Barcelona Open Data, 2017). El portal informativo publicó un total de 101 infográficos, mismos que generaron 334 comentarios y 2,057 reacciones por parte de la audiencia.

A través de la plataforma de Netvizz se adquirieron datos sobre los seguidores y sus preferencias dentro de la página, a lo que prestaron más atención y qué tipo de información compartieron más. Se detectó que el álbum de las trayectorias tuvo el mayor número de interacciones con un total de 4,261 dividido en 335 "me gusta", 134 "me divierte", 95 "me encanta", 12 "me asombra", seis "me enoja", cuatro "me entristece", 199 comentarios, 1,119 compartidos y 2,357 clics en las publicaciones. De igual forma, se encontró que la mayoría de los comentarios en el portal informativo correspondieron a las etiquetas de un usuario a otro con el motivo de compartir con ellos el contenido de la publicación.

Facebook Analytics también brinda datos sobre cada publicación, con esto se lograron determinar las publicaciones con mayor alcance que corresponden a la renuncia de Margarita Zavala con 14,100 personas; ¿sabías qué?: voto en el extranjero con 6,779 personas; las trayectorias de los contendientes con 6,614 personas; y ¿sabías qué?: boletas en casillas especiales con 4,704 personas.

\section{Conclusiones}

Como consecuencia de lo expuesto en el ámbito del desarrollo democrático y concordando con Serrano (2015), los ciudadanos no están informados para emitir decisiones de interés general pese a la falta de transparencia, ya que no hay publicidad sobre las metas alcanzadas durante la administración de los gobernantes. Esta afirmación se contradice con los resultados de la encuesta, los cuales indicaron que los álbumes de las propuestas se ubicaron en el segundo lugar en impacto sobre los seguidores. Sin embargo, las estadísticas de Netvizz señalaron que estas publicaciones fueron algunas de las que cuentan con un menor número de reacciones, compartidos y comentarios. Si bien, parte de la responsabilidad recae en la transparencia de las instituciones, la atención del ciudadano puede que no esté realmente enfocada en la política.

Es posible confirmar teniendo en cuenta la materia de redes sociales con los resultados de Netvizz, mismos que están fuertemente ligados a lo que expresa Salgado (2002), que las redes son un lugar accesible donde se practica la libre expresión y el diálogo de manera más abundante, directa y sin filtro. Lo anterior se respaldó con la existencia de 334 comentarios y 2,057 reacciones en el portal, lo que significa que los seguidores de la página decidieron expresarse. Por otra parte, Facebook (2018) indicó que tener presencia y funcionalidad en la creación y distribución de contenidos en las redes sociales es importante, por medio de la encuesta se confirmó que los seguidores describieron a la página como: "fácil de leer y los gráficos lo hacían entretenido", "muy digerible", "tenía buenos colores, visualmente atractivo, diseño general excelente", "fue muy dinámica y sencilla de entender", entre otros.

En cuanto al tema de la comunicación política, Comorera (2017), al afirmar que las redes sociales se han vuelto indispensables en la vida cotidiana de los ciudadanos, los candidatos y partidos políticos también adoptan estas herramientas tecnológicas con el fin de llegar a sus seguidores, dando paso a una comunicación política relevante. Por tal motivo, las fuentes aplicadas en las publicaciones de las trayectorias, a pesar de que los seguidores en los comentarios los desaprobaban, funcionan como precedentes de información al ser fuente directa del candidato.

Heras y Díaz (2017), señalan que las democracias modernas tienen límites poco explícitos y por lo general varían según las audiencias, esto se atañe al resultado de las encuestas donde se observa que en temática de política, y sobre todo en época electoral, los usuarios de Facebook se ven abrumados 
por el bombardeo de información y, debido a que hay diversos tipos de audiencias, cambia la forma de atraer a todos y cada uno de los subgrupos.

Relacionado específicamente a los jóvenes, Pérez (2017) plantea que no tienen un interés en la materia de política electoral, pues fijan sus metas a corto plazo y no consideran al voto como una fórmula de cambio. No obstante, los resultados proporcionados por los encuestados de 18 a 29 años de edad, revelan que están de acuerdo con pensar que su voto sí tiene un impacto en el cambio de un país y que se mantienen informados sobre cuestiones políticas incluso cuando no es época electoral. Por lo tanto, el perfil del votante puede depender de distintos factores, o simplemente la percepción que se tiene de estar informado y el darle importancia al voto, es diferente según la persona.

En resumen, concordando con Díaz y Muñiz (2016), los ciudadanos recolectan información de portales oficiales, pero también de comentarios de sus conocidos, así como contenido viral en redes sociales, influyendo esto en su ideología política ya que se extiende su panorama de información y posibilidades. En las tablas de contingencia se comprueba que la mayoría de los encuestados afirma conocer aspectos relacionados a la política dado que sus fuentes de información son variadas.

En última instancia, se puede concluir que algunos de los seguidores solo se informaron del proceso electoral en general, esto quiere decir que anteriormente ya tenían una idea de quién sería el contendiente por el cual votarían. Sin embargo, en la segunda encuesta realizada, 14 de 128 de los encuestados respondieron que la página presentaba sesgo a favor de los candidatos, especialmente hacia el contendiente Andrés Manuel López Obrador, por lo tanto, percibían la neutralidad como algo en contra del resto. No obstante, él fue quien más información presentaba a través de sus distintas plataformas de internet y es por eso que fue del que más información se obtuvo para los álbumes de propuestas.

\section{Recomendaciones}

Como parte de este proyecto, se decidieron realizar algunas recomendaciones para enriquecer los futuros proyectos de aquellas personas que deseen crear iniciativas similares. Por lo tanto, la información recabada anteriormente puede tomarse como un precedente para ser utilizadas de manera pertinente.

Primero que nada, es importante verificar los proyectos similares, es decir, crear un benchmark en donde se pueda analizar a la competencia con el fin de descubrir qué es lo que están haciendo los demás, hacer una comparativa en las opciones que se encuentren y conocer sus áreas de oportunidad. De esta forma, se podrá conocer el valor agregado que diferenciará a la nueva iniciativa del resto de las opciones en el campo, una buena opción sería el mencionar las fuentes de donde se obtuvo la información, mismas que deben ser confiables para ser un medio fidedigno ante los usuarios.

Para este proyecto no se realizó un grupo de enfoque en donde se pudiera conocer la opinión de las personas con respecto al nombre del trabajo. Es por ello que se recomienda utilizar esta herramienta con la cual se pueda conocer distintas opiniones o compartir diferentes puntos de vista, con el propósito de considerar aspectos que no se tomaron en cuenta y que con la ayuda de estas nuevas perspectivas guíen al proyecto a obtener resultados más eficaces. Asimismo, en esta misma actividad se podría realizar una consulta sobre qué tan atractivo y fiel es el diseño de la página con la que se va a trabajar.

Por otro lado, si se quiere obtener un mayor alcance en el proyecto, es recomendable utilizar el contenido en diversas plataformas, ya que así se llegará a un público más amplio. Sin embargo, requiere de más tiempo, esfuerzo o un equipo más grande de personas debido a que son más redes sociales y, por lo mismo, el mensaje se debe adecuar al público al que va dirigido. Lo mismo sucede con el enfoque a tratar del proyecto. En este caso se decidió compartir los datos de los candidatos a la presidencia pero se podrían abarcar más categorías a cargos públicos.

Del mismo modo, se sugiere que en la información política que se vaya a compartir, como lo fue la carpeta de “ ¿sabías que?", incremente el número de publicaciones, y así la página tenga una mayor interactividad. Otra manera de enriquecer el proyecto, es buscando una asesoría personalizada durante toda la investigación, ya que así se podrá tener un acompañamiento que ayude a encaminar el objetivo del trabajo. En dado caso que la persona que está asesorando el proyecto no tenga cierta experiencia en una parte específica del trabajo, se puede 
contratar los servicios de un especialista en el área con el fin de potenciar las ideas faltantes.

Es necesario conocer a la audiencia y ver qué tanto han estado reaccionando a las publicaciones con el propósito de verificar los días y horas que tienen más impacto. De esta manera, los seguidores no se sentirán abrumados con tanta información. Del mismo modo, si la iniciativa pertenece al ámbito del emprendimiento, se aconseja realizar un modelo de negocios que afirme que la idea cuenta con las bases necesarias para asegurar que la iniciativa sea realista, alcance sus objetivos y se pueda cumplir en un lapso de tiempo.

Además, se debe fijar un presupuesto con el que se establecerán los gastos monetarios para iniciar el proyecto, ya que así se conocerá si se obtendrán ganancias con la página recuperando o perdiendo posteriormente la inversión o si es necesaria la colaboración de patrocinadores. Partiendo de este punto, es relevante considerar que a veces las publicaciones o imágenes que se quieren promocionar tienen mucho texto $\mathrm{y}$, como consecuencia, las plataformas no permiten que sean publicitadas, como parte de sus políticas, ya que las consideran spam. Por ello es importante que el diseño que se les va a otorgar sea visualmente atractivo, dinámico y sin faltas de ortografía para que así sea más accesible, que sea responsivo al momento de descargar y pueda ser compartido en otras redes sociales.

Se recomienda definir en primera instancia los objetivos del proyecto porque de aquí se derivan las preguntas que respaldarán con la encuesta. Para esto se sugiere asesorarse por medio de un experto en el tema con el propósito de analizar la claridad y viabilidad de los datos. Por ende, el siguiente paso para analizar los resultados de las encuestas es ingresar los datos en la plataforma SPSS para así obtener un mayor provecho de lo recabado, a través de tablas de contingencia.

Por último, cada vez habrá más información sobre el tema, por lo cual hay que estar en constante actualización y leer no solo a través de la web, sino por medio de otras fuentes tales como libros, enciclopedias, documentos oficiales del gobierno, entre otros. Igualmente, se recomienda realizar una gráfica de Gantt con la cual se puedan organizar las actividades y calendarizar los días en los que la información será publicada.

\section{Referencias}

Benítez, R. (2014). Jugando con el grafo social de Facebook en Gephi. Recuperado el 9 de septiembre de 2018 de: http://www.benitezrafa.es/jugando-con-el-grafo-social -de-facebook-en-gephi/

Comorera, J. (2017). Comunicación política y redes sociales. Recuperado el 8 de septiembre de 2018 de: http://rabida .uhu.es/dspace/bitstream/handle/10272/14553/Uso_efe ctividad_y_alcance.pdf?sequence $=2$

Cruz, J. (2016). En picada, el avance del desarrollo democrático en México. Recuperado el 7 de octubre de 2018 de: https://www.proceso.com.mx/440372/en-p icada-avance-del-desarrollo-democratico-en-mexico

Díaz, O. \& Muñiz, C. (2016). Los efectos de la comunicación política en el compromiso político de los jóvenes en la elección presidencial mexicana de 2012. Recuperado el 8 de septiembre de 2018 de: http://www.redalyc.org/ht $\mathrm{ml} / 421 / 42152783008 /$

Facebook. (2018). Anuncios de Facebook. Recuperado el día 30 de agosto de 2018 de: https://www.facebook.com/b usiness/products/ads

Heras, L., Díaz, O. y Medrano, R. (2015). Partidos Políticos, Campañas Electorales y Redes Sociales en lo Local: Elecciones 2015 en el Estado de México. Recuperado el día 7 de septiembre de: https://www.researchgate.net/p ublication/322163068_Comunicacion_politica_campa nas_electorales_y_redes_sociales_en_Mexico

IFE. (2013). Estudio Censal de la Participación Ciudadana en las Elecciones Federales de 2012. Recuperado el 8 de febrero de 2018 de: http://portalanterior.ine.mx/docs /IFE-v2/DECEYEC/DECEYEC-EstudiosInvestigacio nes/InvestigacionIFE/Estudio_Censal_Participacion_C iudadana_2012.pdf

Iniciativa Barcelona Open Data. (2017). Análisis de datos de redes sociales. Recuperado el 9 de septiembre de 2018 de: https://es.slideshare.net/iopendatabcn/curs-27-anlis is-de-datos-de-redes-sociales

La Jornada. (2017). $30 \%$ de los jóvenes representarán el voto del padrón en 2018. Recuperado el 22 de febrero del 2018 de: https://www.vanguardia.com.mx/articulo/repre sentara-el-voto-de-jovenes-30-del-padron-en-2018

Nyczepir, D. (2012). Survey: Youth prefer Facebook GOTV reminder. Recuperado el 13 de febrero de 2018 de: https: //www.campaignsandelections.com/campaign-insider/s urvey-youth-prefer-facebook-gotv-reminder

Pulso D.F. (2015). UNAM lanza plataforma para informar a ciudadanos sobre elecciones. Recuperado el 12 de febrero de 2018 de: http://www.pulsodf.com.mx/unamlanza-plataforma-para-informar-a-ciudadanos-sobreelecciones/ 
Salgado, M. (2002). Marketing Politico: arte y ciencia de la persuasión en democracia. Paidós, Barcelona.

Serrano, A. (2015). La participación ciudadana en México. Recuperado el 3 de octubre de 2018 de: http://www.scie lo.org.mx/scielo.php?script=sci_arttext\&pid=S0185-1 6162015000100005

Sumuano, F. y Nieto, F. (s.f.). Ciudadanía en México ¿Ciudadanía activa? Recuperado el 3 de octubre de 2018 de: https://portalanterior.ine.mx/archivos2/s/DE CEYEC/EducacionCivica/Ciudadania-Activa.pdf

Vázquez, R. (2017). Elecciones y guerra de información digital. Recuperado el 4 de octubre de 2018 de: https: //www.forbes.com.mx/elecciones-y-guerra-de-informa cion-digital/ 